\title{
Perfil lipídico de ovos desidratados com ênfase no seu teor de gorduras trans
}

\author{
Lipid profile of dehydrated eggs with emphasis on the contents of trans fatty acids
}

Silce Adeline Danelon GUASSI ${ }^{1}$, Jocelem Mastrodi SALGADO ${ }^{1 \star}$, Dante Pazzanese Duarte LANNA ${ }^{2}$

\section{Resumo}

O presente trabalho objetivou a determinação do perfil lipídico de ovos integrais desidratados, bem como de gemas desidratadas, a fim de enfatizar seu conteúdo de ácidos graxos de configuração trans. A fração lipídica das amostras foi extraída com hexano/isopropanol e, a seguir, metilada. Os ácidos graxos foram identificados via cromatografia gasosa. Constatou-se que a natureza lipídica dos ovos tem caráter predominantemente insaturado: 63,65\% dos lipídios totais nos ovos integrais e 62,63\% nas gemas. Além disso, foram identificados apenas traços de gorduras trans $(0,24 \%$ nos ovos integrais e $0,27 \%$ nas gemas).

Palavras-chave: lipídios; ovos desidratados; ácidos graxos trans; cromatografia gasosa.

\begin{abstract}
The objective of this work was to determine fatty acids composition in dehydrated eggs and egg yolks emphasizing its contents of trans fatty acids. The fatty acids fraction of the samples was extracted with hexane/isopropanol $(3: 2)$ and then methylated. The profile of the fatty acids was identified by gas chromatography with predominant evidence of insaturated character: 63.65 and $62.63 \%$ of the lipids were unsatureted in the eggs and in the egg yolks, respectively. Traces of trans fatty were also identified $(0.24 \%$ in the hole eggs and $0.27 \%$ in the egg yolks). Keywords: lipids; dry eggs; trans fatty acids; gas chromatography.
\end{abstract}

\section{Introdução}

Ácidos graxos insaturados que contêm ao menos uma dupla ligação de configuração trans são chamados de gorduras trans. Este tipo de configuração pode ocorrer naturalmente, como no caso de alguns produtos lácteos e algumas plantas, mas sua ocorrência está majoritariamente ligada a processos industriais de hidrogenação (VALENZUELA; MORGADO, 1999). Em ovos desidratados, a presença destes compostos ainda não foi identificada de modo direto, embora contenham ácidos graxos insaturados passíveis de conversão.

Atualmente as gorduras trans são motivo de controvérsias e divergências nas mais diversas áreas, incluindo a Bioquímica, a Nutrição e a Epidemiologia, já que são consideradas e reconhecidas como promotoras de desordens funcionais e metabólicas. Seus efeitos negativos estão relacionados à sua estrutura (similar à estrutura de ácidos graxos saturados), ao não conhecimento de suas funções metabólicas específicas e à competição quanto à absorção de ácidos graxos essenciais (VALENZUELA; MORGADO, 1999). Isso significa que as gorduras trans, não só provocam efeitos similares aos provocados por ácidos graxos saturados no organismo, aumentando os teores de LDL (lipoproteínas de baixa densidade) e reduzindo os teores de HDL (lipoproteínas de alta densidade), como também dificultam a absorção de ácidos graxos essenciais, proporcionando um efeito ainda mais catastrófico aos riscos de doenças coronárias (SUBBAIAH; SUBRAMANIAN; LIU, 1998).
Sabe-se ainda, que de acordo com Subbaiah, Subramanian e Liu (1998), o decréscimo da concentração de lipoproteínas de alta densidade estaria relacionado à inibição da atividade enzimática da lecitina-colesterol-acil-transferase, induzindo à formação de ésteres de colesterol saturados, mais aterogênicos. Já o aumento na concentração de moléculas de lipoproteínas de baixa densidade estaria relacionado a desordens metabólicas nos receptores de LDL (HAYASHI et al., 1993).

Contudo, associações entre doenças coronárias e ácidos graxos trans foram observadas pela primeira vez por Willett et al. (1993), o que remete a uma abordagem extremamente recente.

Os ovos são alimentos de alto valor nutricional, já que possuem todas as vitaminas, aminoácidos e minerais essenciais. Sua proteína, a albumina, é dotada de alto valor biológico e foi considerada durante muito tempo como proteína padrão pela Organização para Alimentos e Agricultura da Organização Mundial da Saúde (FAO-OMS) (HARDER, 2005). Atualmente, a OMS estabelece como proteína padrão uma proteína teórica, contudo, a albumina é ainda a proteína que mais se aproxima desta em composição nutricional (VIEIRA, 2000).

Constituem-se ainda como alimentos baratos e acessíveis a populações de todos os níveis sociais (SALVADOR; SANTA, 2002).

Recebido para publicação em 9/3/2007

Aceito para publicação em 19/10/2007 (002350)

'Laboratório de Bromatologia e Nutrição Experimental, Departamento de Agroindústria, Alimentos e Nutrição, Escola Superior de Agricultura "Luiz de Queiroz" -

ESALQ,Universidade de São Paulo - USP, Av. Pádua Dias, 11, CEP 13418-900, Piracicaba - SP, Brasil, E-mail: saguassi@esalq.usp.br, E-mail: jmsalgad@esalq.usp.br*

${ }^{2}$ Laboratório de Nutrição e Crescimento Animal, Departamento de Zootecnia, Escola Superior de Agricultura "Luiz de Queiroz - ESALQ, Universidade de São Paulo - USP,

E-mail: dplanna@esalq.usp.br

${ }^{*}$ A quem a correspondência deve ser enviada 
O presente trabalho objetivou, portanto, a determinação do perfil lipídico de ovos integrais desidratados, bem como de gemas desidratadas, a fim de enfatizar seu conteúdo de ácidos graxos de configuração trans.

\section{Material e métodos}

Todos os procedimentos foram realizados na Escola Superior de Agricultura "Luiz de Queiroz" - ESALQ, campus da Universidade de São Paulo - USP, na cidade de Piracicaba. As análises do conteúdo de lipídios de configuração isomérica trans das amostras foram realizadas por cromatografia gasosa nos Laboratórios de Nutrição e Crescimento Animal, do Departamento de Zootecnia e de Toxicologia de Inseticidas e Resíduos de Pesticidas, do Departamento de Entomologia, Fitopatologia e Zoologia Agrícola.

As amostras de ovos foram desidratadas pelo processo de spray dryer e foram fornecidas pela Izumi Indústria e Comércio Ltda (CNPJ: 04.563.101/0001-21). A extração da fração lipídica e metilação das amostras foi efetuada com base em Hara e Radim (1978) com adaptações realizadas em decorrência da composição lipídica dos ovos desidratados.

\subsection{Extração da fração lipídica}

Para a extração da fração lipídica dos ovos desidratados, foram pesadas e homogeneizadas, em vórtex, por 60 segundos, $2 \mathrm{~g}$ das amostras em tubo de ensaio juntamente com $28 \mathrm{~mL}$ de solução hexano/isopropanol (3:2). As soluções foram deixadas em descanso até que a parte sólida se depositasse no fundo dos tubos. O conteúdo dos tubos foi então filtrado em papel de filtro sob vácuo em kitassato. Para a lavagem e limpeza do funil de vidro entre as filtragens, utilizou-se hexano. Os conteúdos dos kitassatos foram posteriormente transferidos para tubos de ensaios de $50 \mathrm{~mL}$ com o acréscimo de aproximadamente $2 \mathrm{~g}$ de carvão vegetal, para a retenção de pigmentos que pudessem vir a danificar a coluna do cromatógrafo. Foram também adicionados $12 \mathrm{~mL}$ de sulfato de sódio para a separação entre hexano e isopropanol. As soluções foram homogeneizadas em vórtex por 30 segundos e então descansaram por 10 minutos para a separação entre as fases.

O sobrenadante dos tubos, contendo as frações lipídicas das amostras, foi transferido para tubos de extração contendo $1 \mathrm{~g}$ de sulfato de sódio cada. Nestes tubos o nitrogênio foi insuflado por 30 segundos e as soluções permaneceram em descanso por mais 30 minutos.

O sobrenadante dos tubos de extração (composto pela fração lipídica mais hexano) foi ainda transferido para frascos âmbar que foram colocados em banho-maria pré-aquecido a $40^{\circ} \mathrm{C}$. O hexano foi evaporado sob nitrogênio até que apenas a gordura permanecesse e fosse por fim insuflada com nitrogênio e armazenada a $-20^{\circ} \mathrm{C}$, para posterior metilação.

\subsection{Metilação}

Soluções de metilação $(1,75 \mathrm{~mL}$ de $\mathrm{MeOH}$ e $0,4 \mathrm{~mL}$ de $\mathrm{NaOMe}$ ) e de terminação ( $1 \mathrm{~g}$ de ácido oxálico colocado em estufa, a $120^{\circ} \mathrm{C}$, por 30 minutos, resfriado em dessecador e adicionado de $30 \mathrm{~mL}$ de dietil éter) foram previamente preparadas.

As amostras foram descongeladas e o hexano foi evaporado sob nitrogênio, enquanto as amostras permaneceram em placa de aquecimento sob temperatura necessariamente inferior a $40^{\circ} \mathrm{C}$. Foram pesados $40 \mathrm{mg}$ da alíquota restante, adicionados de $2 \mathrm{~mL}$ de hexano e $40 \mu \mathrm{L}$ de metil acetato, sendo homogeneizados por 30 segundos. Ressalta-se que o metil acetato é usado para minimizar os efeitos da saponificação, já que as reações secundárias são direcionadas para o metil acetato e não para os ácidos graxos das amostras.

A estas soluções foram adicionados $40 \mu \mathrm{L}$ da soluçaão de metilação, sendo homogeneizadas por 2 minutos e deixadas em descanso. Foram adicionados $60 \mu \mathrm{L}$ da solução de terminação e as soluções foram novamente homogeneizadas por 30 segundos. Foram também adicionados $200 \mathrm{mg}$ de cloreto de cálcio e as soluções descansaram por 1 hora para a remoção do metanol, prevenindo problemas com o Pico do Solvente e evitando a deterioração da coluna do cromatógrafo.

As soluções foram centrifugadas a $3200 \mathrm{rpm}$, durante 5 minutos, a $5^{\circ} \mathrm{C}$. Os sobrenadantes foram por fim transferidos para recipientes específicos (GLC) que foram acondicionados em freezer para uso futuro.

\subsection{Obtenção do perfil lipídico das amostras por cromatografia gasosa}

O perfil dos ácidos graxos foi determinado através de cromatografia gasosa (com cromatógrafo ThermoFinnigan ${ }^{\circ}$, modelo Focus - São Paulo, Brasil), utilizando-se uma coluna capilar de sílica fundida CP-SIL 88 ( $100 \mathrm{~m} \times 0,25 \mathrm{~mm} \times 0,2 \mathrm{~mm}$, Varian) e um detector de ionização de chama (FID). Foi utilizado também um programa de gradiente de temperatura, no qual o tempo total da corrida foi de 70 minutos, a temperatura do injetor foi de $250{ }^{\circ} \mathrm{C}$, e a do detector de $300{ }^{\circ} \mathrm{C}$.

A injeção foi no modo "split" com relação 10:1. O nitrogênio foi utilizado como gás de arraste, com fluxo de $1,8 \mathrm{~mL}$ por minuto e 18 psi de pressão na cabeça da coluna.

O perfil dos ácidos graxos foi expresso em porcentagem total. Para isso, foi utilizado o CRM-14 (Comission of the European Communities, Comunity Bureau of Reference, Bruxelas, Bélgica), que tem valores certificados para onze ácidos graxos usados para estabelecer os fatores de correção para cada um dos ácidos graxos certificados que foram utilizados para transformar o Pico em porcentagem de área.

\section{Resultados e discussão}

A Tabela 1 mostra os perfis lipídicos de amostras de ovos integrais pasteurizados desidratados e de gemas pasteurizadas desidratadas, identificados pela coluna.

Nota-se que ambos os perfis lipídicos mostraram naturezas predominantemente insaturadas, tanto para os ovos integrais quanto para as gemas.

Os ácidos graxos insaturados corresponderam a $63,65 \%$ dos lipídios totais nos ovos integrais e a $62,63 \%$ nas gemas, sendo 
Tabela 1. Perfil lipídico de amostras de ovos integrais pasteurizados desidratados e gemas pasteurizadas desidratadas.

\begin{tabular}{|c|c|c|c|c|}
\hline & \multicolumn{2}{|c|}{ Ovos Integrais } & \multicolumn{2}{|c|}{ Gemas } \\
\hline & Ácido graxo & Área percentual & Ácido graxo & Área percentual \\
\hline Ácido palmítico & $\mathrm{C} 16: 0$ & 25,15 & C16:0 & 26,27 \\
\hline Ácido heptadecanóico & C17:0 ISO & 0,76 & C17:0 ISO & 0,79 \\
\hline Ácido palmitoleico & C16:1 C9 & 2,84 & C16:1 C9 & 2,91 \\
\hline Ácido heptadecanóico & $\mathrm{C} 17: 0$ & 0,19 & $\mathrm{C} 17: 0$ & 0,14 \\
\hline Ácido esteárico & C18:0 & 7,67 & C18:0 & 7,88 \\
\hline Ácido oleico & C18:1 T11 & 0,13 & C18:1 T11 & 0,15 \\
\hline Ácido oleico & C18:1 C9 & 41,08 & C18:1 C9 & 43,13 \\
\hline Ácido oleico & C18:1 C11 & 1,82 & C18:1 C11 & 1,97 \\
\hline Ácido oleico & 18:1 C15 & - & 18:1 C15 & - \\
\hline Ácido oleico & $\mathrm{C} 18: 1 \mathrm{C} 13$ & 0,04 & $\mathrm{C} 18: 1 \mathrm{C} 13$ & 0,04 \\
\hline Ácido linoleico & $18: 2$ C9 C12 & 14,06 & 18:2 C9 C12 & 11,42 \\
\hline Ácido linolênico & $18: 3$ & 0,09 & $18: 3$ & 0,06 \\
\hline Ácido eicosenóico & $20: 1$ & 0,43 & $20: 1$ & 0,37 \\
\hline Ácido linoleico & 18:2 C9 T11 & 0,09 & 18:2 C9 T 11 & 0,11 \\
\hline Ácido linoleico & 18:2 T10 C12 & - & 18:2 T10 C12 & - \\
\hline Ácido behenico & $22: 0$ & 0,09 & $22: 0$ & 0,05 \\
\hline Ácido lignocérico & $24: 0$ & 1,16 & $24: 0$ & 1,29 \\
\hline Ácido araquidônico & $20: 4$ & 0,10 & $20: 4$ & 0,11 \\
\hline Ácido docosadienóico & $22: 2$ & 0,29 & $22: 2$ & 0,35 \\
\hline Ácido eicosapentaenóico & $20: 5$ & 0,26 & $20: 5$ & 0,29 \\
\hline \multirow[t]{2}{*}{ Ácido docosahexaenóico } & $22: 6$ & 0,09 & $22: 6$ & 0,15 \\
\hline & Total & 96,42 & Total & 97,55 \\
\hline
\end{tabular}

48,08 e 49,80\% monoinsaturados, e 15,56 e $12,83 \%$ poliinsaturados, respectivamente.

A porcentagem de ácidos graxos saturados, equivalente a $36,34 \%$ nos ovos integrais, teve um acréscimo quando somente as gemas foram consideradas (37,36\%), e ainda assim esse percentual correspondeu a pouco mais que a terça parte da fração lipídica analisada.

Já a porcentagem de ácidos graxos trans encontrada nas amostras, principal ênfase deste trabalho, pôde ser considerada como apenas um traço da fração lipídica, já que no caso dos ovos integrais foi equivalente a $0,24 \%$, enquanto que nas gemas foi de $0,27 \%$.

Constatações a respeito de ácidos graxos de configuração trans em ovos desidratados não foram encontradas na literatura, e este tipo de resultado torna-se dessa forma inédito do ponto de vista das análises do perfil lipídico em ovos. Guardiola et al. (1994), entretanto, analisaram ovos frescos e identificaram um percentual de gorduras trans equivalente a $0,7 \%$. De acordo com os autores, o conteúdo de gorduras trans nos ovos frescos estaria relacionado ao teor destes ácidos graxos na dieta das aves. A partir desta comparação, pode-se supor que o processo de desidratação ao qual os ovos foram submetidos não representou uma variável significativa para a elevação do teor de gorduras trans encontrado, o que pode ser considerado um importante fator para a agregação de valor comercial a este tipo de processo.

A natureza predominantemente insaturada dos lipídios encontrados em ovos, contudo, pôde ser constatada por diversos outros autores, como Noble, Cocchi e Turchetto (1990), que identificaram um percentual de ácidos graxos monoinsaturados equivalente a $46 \%$, aproximadamente, em amostras de gema.
Rodrigues et al. (2005) teriam identificado valores semelhantes, correspondentes a 32,96\% para ácidos graxos saturados e $63,71 \%$ para ácidos graxos insaturados. Ainda em amostras de gema, Pita et al. (2004) identificaram valores de 27,47\% para ácidos graxos saturados, 52,57\% para ácidos graxos monoinsaturados e 19,95\% para ácidos graxos poliinsaturados.

\section{Conclusões}

Constatou-se que o perfil lipídico dos ovos integrais e gemas analisado apresentou natureza predominantemente insaturada. Foram identificados ainda traços de ácidos graxos de configuração trans equivalentes a 0,24 e $0,27 \%$, respectivamente.

Esses resultados mostram que o processo de desidratação ao qual os ovos foram submetidos pode agregar valor comercial ao produto e conseqüentemente benefícios à saúde do consumidor.

\section{Agradecimentos}

Nossos mais sinceros agradecimentos ao Conselho Nacional de Desenvolvimento Científico e Tecnológico (CNPq), à Fundação de Amparo à Pesquisa do Estado de São Paulo (FAPESP), à empresa Izumi Indústria e Comércio Ltda. e ao Prof. Dr. Carlos Holger Wenzel Flechtmann.

\section{Referências bibliográficas}

GUARDIOLA, F. et al. Fatty Acid Composition and Nutritional Value of Fresh Eggs, from Large- and Small-Scale Farms. J. Food Compos. Anal., Orlando, Fla., v. 7, n. 3, p. 171-188, 1994. 
HARA, A.; RADIN, N. S. Lipid Extraction of Tissues with Low-toxicity Solvent. Anal. Biochem., University of Bologna, Cesena, Italy, v. 90, n. 1, p .420-426, 1978.

HARDER, M. N. S. Efeito do urucum (Bixa orellana) na alteração de características de ovos de galinha poedeiras. 2005, 74 p. Dissertação (Mestre em Ciência e Tecnologia de Alimentos) - Escola Superior de Agricultura "Luiz de Queiroz", Universidade de São Paulo (USP), Piracicaba, 2005.

HAYASHI, K. et al. Effect of dietary hydrogenated corn oil (transoctadecenoate rich oil) on plasma and hepatic cholesterol metabolism in the hamster. Atherosclerosis, v. 99, n. 1, p.97-106, 1993.

NOBLE, R. C.; COCCHI, M.; TURCHETTO, E. Egg fat: a case for concern? World's Poult. Sci. J., v. 46, n. 2, p. 109-118, 1990.

PITA, M. C. G. et al. Efeito da Adição de Ácidos Graxos Insaturados e de Vitamina E à Dieta de Galinhas e seu Reflexo na Composição Lipídica e Incorporação de $\alpha$-Tocoferol na Gema do Ovo. Braz. J. Vet. Res. Anim. Sci., São Paulo - SP, v. 41, n. 1, p. 25-31, 2004.
RODRIGUES, E. A. et al. Desempenho, Qualidade da Casca e Perfil Lipídico de Gemas de Ovos de Poedeiras Comerciais Alimentadas com Níveis Crescentes de Óleo de Soja no Segundo Ciclo de Postura. Acta Sci. Anim. Sci., Maringá, v. 27, n. 2, p. 207-212, 2005.

SALVADOR, M.; SANTA, P. D. Teores de macronutrientes e de colesterol em dierentes tipos de ovos. Boletim CEPPA, Curitiba, v. 20, n. 1, p. 133-140, 2002.

SUBBAIAH, P. V.; SUBRAMANIAN, V. S.; LIU, M. Trans unsaturated fatty acids inhibit lecithin: cholesterol acyltransferase and alter its positional specificity. J. Lipid Res., v. 39, n. 7, p. 1438-1447, 1998.

VALENZUELA, A.; MORGADO, N. Trans Fatty Acid Isomers in Human Health and in the Food Industry. Biol. Res., Santiago, v. 32, n. 4, p. 273-287, 1999.

VIEIRA, E. C. Os Valores do Ovo. Avicultura Industrial, Campinas, v. 90, n. 1076, p. 17-19, 2000.

WILLETT, W. C. et al. Intake of trans fatty acids and risk of coronary heart disease among women. Lancet., v. 341 , n. 8845, p. 581-585, 1993. 